\title{
APLICACIÓN DE LA FOTOGRAMETRÍA ARQUITECTURAL DIGITAL EN LA CONSERVACIÓN DE INMUEBLES PATRIMONIALES
}

\section{APPLICATION OF DIGITAL ARCHITECTURAL PHOTOGRAMMETRY ON CONSERVATION OF HERITAGE BUILDINGS.}

FECHA DE RECIBO: OCTUBRE 31, 2012

FECHA DE ACEPTACIÓN: DICIEMBRE 20, 2012

\begin{abstract}
ANA LUISA RODRÍGUEZ QUESADA/ Doctora en Ciencias Técnicas. Profesora Titular de la Facultad de Ingeniería. Universidad Holguín, Cuba • FRANK NAVARRO TAMAYO/ Doctor en Ciencias Técnicas. Profesor Titular de la Facultad de Ingeniería. Universidad Holguín, Cuba - VERÓNICA ÁVILA AYÓN/ Doctora en Ciencias Técnicas. Profesora Titular de la Facultad de Ingeniería. Universidad Holguín, Cuba - ANGÉLICA MARÍA RODRÍGUEZ CONCEPCIÓN/ Estudiante de Ingeniería Civil de la Universidad de Holguín, Cuba - FÁTIMA MARTíNEZ HERNÁNDEZ/ Estudiante de Ingeniería Civil de la Universidad de Holguín, Cuba • MARÍA BÁRBARA DOIMEADIOS HERNÁNDEZ/ Estudiante de Ingeniería Civil de la Universidad de Holguín, Cuba • VIOLETA SALGADO FERNÁNDEZ, Estudiante de Ingeniería Civil de la Universidad de Holguín, Cuba • ALEXANDER JUSTEL BETANCOURT/ Estudiante de Ingeniería Civil de la Universidad de Holguín, Cuba
\end{abstract}

\section{RESUMEN:}

Este artículo sintetiza los aspectos fundamentales de la investigación sobre la aplicación de la fotogrametría digital con el fin de mantener y/o recuperar los valores del patrimonio histórico de una nación, estimulando la conciencia de realizar remodelaciones en las obras arquitectónicas para su conservación, previendo catalogar cada época, para conformar un registro fotográfico que permita con posterioridad tratamientos de restauración o reconstrucción de inmuebles (medición, levantamiento, creación de planos y otros detalles), siguiendo los lineamientos de rigurosidad propuestos al respecto por los principales organismos nacionales e internacionales: Consejo Internacional de Monumentos y Sitios, Comité Internacional de Fotogrametría Arquitectural. El trabajo tiene el objetivo de proponer una técnica simple de fotogrametría en combinación con Sistemas de Información Geográfica SIG para realizar acciones de conservación del patrimonio edificado del casco histórico de la ciudad de Holguín, Cuba. Se propone la cámara digital como herramienta para este tipo de levantamientos, exponiendo sus características más importantes para tal fin.

\section{PALABRAS CLAVE:}

Fotogrametría digital, conservación de inmuebles con valores patrimoniales

\section{ABSTRACT:}

This article concerns the application of digital photogrammetry in order to maintain and/or restore historic heritage valuesof a nation, stimulating awareness of perform ingarchitectural remodeling works for conservation, providing catalog every time, to form A photographic record after allowing restoration treatments or reconstruction of buildings (measuring, surveying, building plans and other details), following stringent guidelines proposed in this regard by leading national and international organizations: International Council on Monuments and Sites, International Committee of Architectural Photogrammetry. This work aims proposing a simple technique of photogrammetry in combination with Geographic Information Systems (GIS) to conservation actions of the historic of Holguin city's built heritage, Cuba. Digital camera is proposed as a tool for this type of survey, giving the most important features for this purpose.

\section{KEYWORDS:}

Digital Photogrammetry, conservation properties, heritage values 
a ciudad de Holguín, Cuba, cuenta con una gran cantidad de construcciones y monumentos declarados como patrimonio histórico, registrados en el municipio en un sistema de inventario convencional (Registro gráfico, sin soporte digital). Por este motivo, su actualización ha sido deficiente y data de años atrás.

En el trabajo objeto de investigación que aquí se presenta, el principal enfoque está en la fusión entre la historia del arte y la tecnología, para posibilitar un mayor respaldo a lo que se considera imprescindible para la historia de una nación, o sea, la preservación de su patrimonio histórico. El fundamento del trabajo consistió en definir un método para el uso de la restitución fotogramétrica y los Sistemas de Información Geográfica -SIGcon el propósito de lograr datos que, con un elevado grado de precisión, permitieran realizar análisis comparativos en información dimensional y ejecutar planos, secciones y elevaciones y detalles de edificios históricos, objetos arqueológicos y otros elementos de valor en ciudades que turísticas, lo cual, según ESRI (1996), contribuirá a la preservación, difusión y estímulo de la consulta de la memoria histórica de la ciudad como fuente de sustento de los procesos de conservación.

En los países de América Latina y el Caribe, los niveles de desarrollo de la conservación de monumentos aplicando tecnologías de punta son dispares y atraviesan, en la mayoría de los casos, una etapa inicial de organización de la información y fortalecimiento institucional. Según Bähr (1991), las competencias colectivas en torno al conjunto de especializaciones en los campos de procesamiento digital de imágenes, modelación y desarrollo sustentable, potenciarán los resultados de este trabajo en virtud de generalizar tecnologías científicamente soportadas para apoyar la toma de decisiones en el contexto de desarrollo de un turismo sostenible.

La cumbre mundial sobre desarrollo sostenible, celebrada en Johannesburgo en 2002, se pronunció en relación con las iniciativas de Latinoamérica y el Caribe, reconociendo la importancia de acciones regionales en esta dirección, teniendo en cuenta las singularidades de la región, las visiones compartidas y la diversidad cultural y abogando por la adopción de acciones concretas en las diferentes áreas de desarrollo sostenible.

El Consejo Internacional de Monumentos y Sitios (ICOMOS) recomienda que cada país constituya un archivo fotogramétrico de sus monumentos históricos. La utilización de la Fotogrametría Arquitectural Digital (FAD), aliada a bancos de datos que contengan información sobre los monumentos, según ha sido planteado por Kreiling, (1976), es uno de los más importantes instrumentos tecnológicos disponibles para el registro y para la preservación de los monumentos históricos; lo anterior coincide con la apreciación de Cheli (1996), en el sentido de que estos son la historia viva de la nación.

Sin embargo, en la actualidad la fotogrametría es poco usada en América Latina para la preservación del patrimonio histórico nacional, entre otras razones por el alto costo de los equipos que requiere y por el poco conocimiento por los especialistas de la conservación, a pesar de que los países que forman este continente tienen una gran riqueza arqueológica, que incentiva el turismo cultural, lo que refuerza la necesidad de introducir los avances de la ciencia y la tecnología en aras de conservar para las generaciones futuras el patrimonio histórico de estas naciones, con la participación activa de todos los sectores, respetando los derechos económicos, sociales y culturales de estas comunidades.

Según Buill, Núñez \& Rodríguez (2007), la Fotogrametría Arquitectural Digital (FAD) empieza a aplicarse en el mundo a mediados de la década de los 90`scon el liderazgo de EUA, Alemania y Reino Unido, entre otros. Hacia el año 2000, los países de América Latina y el Caribe comienzan a insertarse en estas iniciativas, aunque orientadas generalmente a su introducción desde el punto de vista teórico.

Este trabajo se propone partir de la formalización y modelación de los escenarios de utilización para derivar en la aplicación de los avances internacionales en función de responder a las realidades de Cuba y, específicamente, del municipio de Holguín como ciudad de referencia para su generalización en el ámbito nacional. El principal aporte se basa en la integración de las experiencias de varios países de la región en torno a la FAD y el desarrollo de modelos prácticos de bajo costo para su aplicación, aprovechando las fortalezas nacionales y del municipio complementarias en la difusión de modelos conceptuales, metodologías y planes de implementación útiles para todos, como se sugiere por Navarro \& Rodríguez \& Ávila \&Loch (2010), en particular para enfrentar de manera más efectiva la aplicación a escala de ciudad de los retos que impone el desarrollo de las acciones de conservación.

Como problema de investigación, se identificaron las deficiencias en la estrategia de conservación de los bienes inmuebles en el casco histórico de la ciudad de Holguín; en dirección a lo cual se propuso, como objetivo general de la misma, la propuesta de una técnica simple de aplicación de la fotogrametría arquitectural digital en combinación con SIG para conservar las construcciones con valores patrimoniales del centro histórico de la ciudad de Holguín, valorando la sostenibilidad de esta técnica para prevenir impactos y favorecer la toma de conciencia por los estudiantes de Ingeniería Civil sobre el uso de recursos con una tecnología adecuada al desarrollo local.

Así mismo, la investigación planteó, como sus objetivos específicos: Introducir la FAD como herramienta de última generación, para el tratamiento de imágenes y su aplicación en la conservación de bienes inmuebles; crear una base de datos en la cual se puedan obtener imágenes con excelente calidad y medidas exactas de construcciones insignias de la ciudad de Holguín; y elaborar un modelo para la calibración de líneas de la cámara digital no profesional para la toma de fotos por la $F A D$, así como un procedimiento para el procesamiento digital 
de imágenes de bienes inmuebles con valores reconocidos del caso histórico de la ciudad de Holguín.

\section{METODOLOGÍA DE TRABAJO}

La investigación se estructuró como un proyecto multidisciplinar destinado a la confección de la documentación monumental de la ciudad de Holguín, para lo cual se integraron diferentes profesionales con los objetivos de obtener una visión más amplia del patrimonio de la ciudad; con esta cooperación entre diferentes especialidades se espera, como beneficio colateral, una mejora de la formación profesional, ya que estos temas no están incluidos en el plan de estudio vigente para la carrera de Ingeniería Civil de la Universidad de Holguín.

- La metodología de trabajo incluyó las siguientes actividades:

- Caracterización de deterioros en la edificación seleccionada.

- Tratamiento digital de las imágenes para la caracterización y control de datos.

- Determinación de las causas principales de deterioro.

- Propuesta de medidas.

- Ejecución de medidas.

En el trabajo de investigación se aporta una técnica para realizar acciones de conservación del patrimonio edificado del casco histórico de la ciudad de Holguín, Cuba, la cual fue presentada por los autores en el 5th International Structural Engineering and Construction Conference, Challenges, Opportunities and Solutions in Structural Engineering and Construction realizado en Los Angeles, USA (Navarro et al, 2010). En dicha técnica se propone la cámara digital como herramienta para este tipo de levantamientos, exponiendo sus características más importantes para tal fin.

\section{APLICACIONES DE LA FOTOGRAMETRÍA COMO TECNOLOGÍA}

La fotogrametría, como herramienta tecnológica, se aplica para la conservación de edificaciones, además en la ortopedia, criminalística, topografía, viales y medioambiente e investigaciones subacuáticas. Ver Gráfica 1

\section{TÉCNICAS FOTOGRAMÉTRICAS}

La Carta de Venecia define la autenticidad histórica como un valor fundamental para garantizar la objetividad de los trabajos de restauración y alienta la importancia de los levantamientos exactos y precisos de los monumentos en determinada época, propuesta por Menéndez (1987) para preservar los

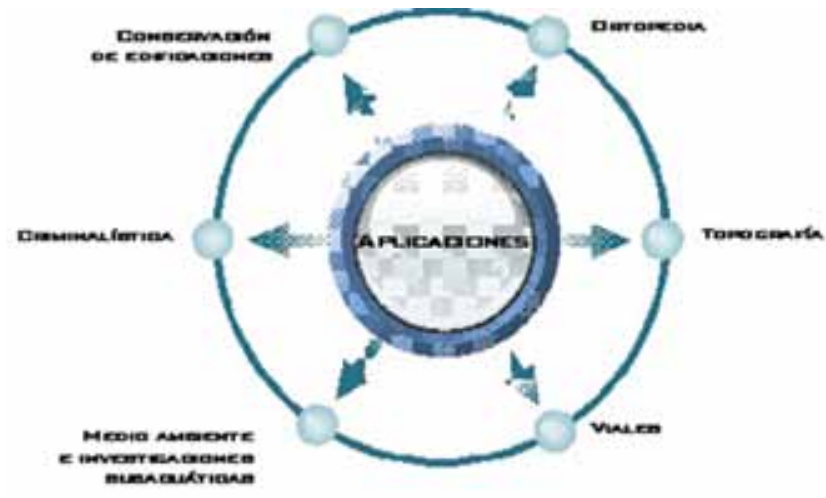

Fuente: Elaboración propia

Gráfica 1. Aplicaciones de la fotogrametría

detalles estéticos, técnicos o históricos. De ahí la importancia de la fotogrametría digital, la cual, como afirma Altrock (2004), genera resultados más rápidos y precisos, tornándose posible obtener precisiones milimétricas en las medidas siendo de gran importancia para guardar documentación científica de bienes culturales, así como para el análisis y control de deformaciones en edificaciones.

A partir de 1950,el avance en la aplicación de la fotogrametría se ve favorecido con el desarrollo de los ordenadores electrónicos, que no pasó inadvertido para encargados de la protección del patrimonio, que habían evolucionado a su vez hacia métodos de mayor rigor para sustentar los principios de la conservación, tal como refieren Cela \& Paredi (1996), Mena (1992).

Es así como, en 1968, tres años después de su constitución, afirma Piechel (1985),el Consejo Internacional de Monumentos y Sitios (ICOMOS) organizó en París el 1er. Simposio Internacional, dedicado a la fotogrametría arquitectónica. Como resultado de las Resoluciones aprobadas en éste, fue creado en 1970 el Comité Internacional para la Fotogrametría Arquitectónica (CIFA) y actualmente trabaja como grupo permanente en la Sociedad Internacional de Fotogrametría y Teledetección (ISPRS).

En Cuba, el empleo de la fotografía para el estudio de las construcciones arquitectónicas, se inicia a finales de la década del 70 por el Instituto Cubano de Geodesia y Cartografía (ICGC), dirigidos fundamentalmente a la conservación y restauración de monumentos en la Habana Vieja, así como en las ciudades de Trinidad y Santiago de Cuba.

La tarea fundamental de la fotogrametría, plantea Claus (1983), es establecer una relación geométrica entre la imagen y el objeto como este existía en el momento de la toma fotográfica. Una vez establecida correctamente esta relación, es posible realizar una restitución fotogramétrica o sea, obtener una representación gráfica o numérica de un objeto fotografiado, relación ésta que puede ser clasificada en tres categorías: 
- Gráfica: usando las relaciones geométricas

- Analógica: usando componentes óptico-mecánicos

- Analítico/digital: usando modelo matemático y procesamiento digital.

La fotogrametría digital, comenta Santa Cruz (2013), tornó la restitución fotogramétrica más simple, flexible y accesible, permitiendo una variedad de aplicaciones, una vez que el procesamiento es realizado por microcomputadores a través de programas específicos de fotogrametría, asociados a otros equipamientos de uso general, como scanner y diversos tipos de cámaras. En esta investigación, se describen también los procedimientos adoptados y los resultados obtenidos en el registro de edificaciones arquitectónicas a partir de la utilización del software fotogramétrico digital Photo Modeler,el cual emplea las técnicas de monorestitución y la restitución a partir de varias fotografías. El Photo Modeler permite obtener varios productos a partir de imágenes digitales: coordenadas de puntos, distancias, modelos en 3D, modelos tridimensionales con la textura original del objeto, ortofotos, además de que posibilita la exportación de los resultados en diversos formatos.

\section{MONORRESTITUCIÓN}

La monorrestitución, según lo planteada por Leão de Amorim. \& Groetelaars (2004), presupone la solución del problema a través de una única fotografía, siendo necesarias las informaciones sobre aspectos geométricos del objeto, ya que en una fotografía, las coordenadas de un punto, pueden corresponder a infinidad de ellas en el espacio del objeto. Para resolver este problema es necesario acudir a otras informaciones como paralelismo, perpendicularidad de sus aristas e identificación de sus ejes $X, Y, Z$ con la posición de la cámara en el momento de ser tomada la fotografía. La determinación de la escala requiere conocer, por lo menos, una dimensión del objeto, así pueden clasificarse en tres tipos los objetos que pueden ser restituidos a partir de una única fotografía: objetos planos, objetos planos irregulares y objetos donde es posible la identificación de sus tres ejes.

La monorrestitución también permite obtener las dimensiones de un objeto a partir de otros programas menos complejos como DigiCAD 3D y RolleiMetric, los cuales realizan la rectificación de la fotografía cuando son conocidas las coordenadas de dos vértices de la superficie. Luego de rectificar las fotos, éstas pueden ser exportadas a un editor de diseño para el trazado de sus características geométricas. Este proceso posee limitaciones prácticas pues solo pueden ser restituidas las partes visibles en la fotografía, no obstante es una técnica de bajo costo, de fácil utilización y rapidez. Se recomienda su aplicación en la restitución de monumentos que no existen y de los cuales se conservan solo fotografías.

\section{RESTITUCIÓN A PARTIR DE VARIAS FOTOGRAFÍAS.}

Con esta técnica, según describen Leão de Amorim. \& Groetelaars (2004), los objetos son fotografiados en diversas posiciones y cada parte del objeto debe ser fotografiada más de una vez con puntos superpuestos. A partir de la identificación de los puntos homólogos o comunes entre varias fotografías, es posible realizar las intersecciones para la restitución del objeto. Este fue el primer tipo de restitución usado desde los principios de aplicación de la fotogrametría, antecede a los métodos citados anteriormente. Fue utilizado en su forma inicial, a través de procesos gráficos de medición y representación "punto por punto". Este método presentaba limitaciones en cuanto a rapidez y precisión.

Facon (1993) recuerda que, con los avances tecnológicos y el aumento de la capacidad computacional, fue posible utilizar los principios y fundamentos de esta técnica (método gráfico) para la creación de programas capaces de solucionar los problemas analíticamente, a través de ecuaciones matemáticas.

La utilización de esta técnica crece cada día, principalmente cuando se trata de levantamientos arquitectónicos, debido principalmente a factores como los siguientes:

- Utilización de hardware de uso general, como scanner, cámaras fotográficas, comunes o digitales y microcomputadores asociados a programas específicos.

- Diferentes distancias focales y diversos ángulos, permitiendo el levantamiento fotográfico de forma que abarque toda la edificación.

- Reducción del número de puntos de control.

- Variedad de productos a obtener: modelos foto-realísticos, ortofotos, diseños en ambiente computacional, posibilitando la integración de los archivos en diversos programas.

- Mediciones y parámetros desconocidos son calculados analíticamente, o sea no es necesario conocer los parámetros de la cámara, ni su posición u orientación en el momento de la toma de la fotografía.

- Posibilidad de detección de errores.

A pesar de todas las ventajas de esta método, debido a la ausencia de visión estereoscópica la restitución a partir de varias fotografías esta limitada a la determinación de puntos discretos que son fácilmente identificables en diversas imágenes, no siendo suficiente en algunos casos, especialmente si el objeto presenta formas geométricas que pueden ser bien definidas a través de puntos y líneas, formas irregulares o curvas, como esculturas y detalles arquitectónicos. 
ARTE \& DISEÑO, ISSN: 1692-8555, Vol. $11 \mathrm{~N}^{\circ} 1$, Enero - Junio 2013

Aplicación de la Fotogrametría Arquitectural digital en la conservación de Inmuebles patrimoniales, págs. 5-11

\section{CASO DE ESTUDIO: REGISTRO DE UNA EDIFICACIÓN EN EL CENTRO HISTÓRICO DE LA CIUDAD DE HOLGUÍN}

\section{METODOLOGÍA APLICADA}

La investigación emprendida debía brindar información sobre una serie de aspectos básicos, destacándose entre ellos:

- Tipos de daños en los objetos estudiados

- Magnitud, distribución y forma de presentación de los daños

- Factores causantes de los daños

- Características óptimas de las imágenes para el registro de cada tipo de desperfecto.

Para ello se siguió el método de trabajo esquematizado en la gráfica a continuación.

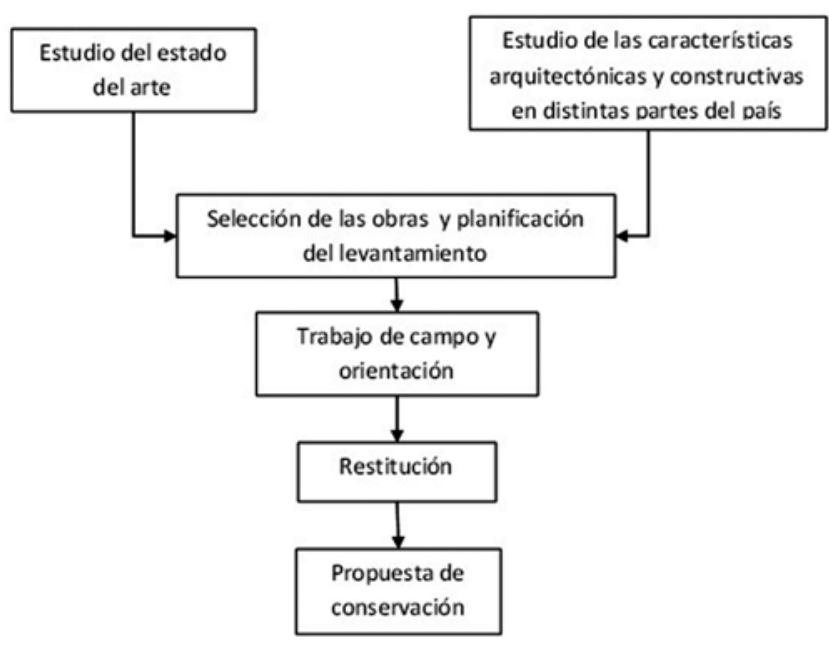

Gráfica 2. Método de trabajo

\section{EQUIPAMIENTO Y METODOLOGÍA SEGUIDA PARA LA TÉCNICA PROPUESTA}

Con base en los fundamentos teóricos de la investigación, se busca una tecnología adecuada a las condiciones socioeconómicas y ambientales del contexto para proponer una técnica simple de aplicación de la fotogrametría arquitectural digital en combinación con un SIG, para conservar las cons-trucciones con valores patrimoniales del centro histórico de la ciudad de Holguín.

Aquí, se deben desarrollar dos líneas distintas de trabajo, las cuales se implementaron en desarrollo paralelo: la primera de ellas implica la recopilación de la información existente de tipo documental y el levantamiento físico con cámaras nométricas y técnicas alternativas de fotogrametría (condición ésta previamente impuesta por el costo del equipamiento y la decisión de emplear técnicas de uso simple que permitan que personal con cierto grado de capacitación realicen el levantamiento); la segunda línea de trabajo se dedicó a la carga de la información en un SIG, la cual permitió el análisis de la información almacenada y la elaboración de cartografía afín.

Para ello se tuvo en cuenta la información catastral del municipio de Holguín, consistente en datos de ubicación, propietario, año de construcción, uso, constructor, grado de interés histórico, paisajístico y arquitectónico, además de sus datos de identificación catastral (partida, circunscripción, sección, manzana, parcela, etc.). Para el levantamiento fotográfico, se empleó una cámara digital de alta resolución (10 megapíxeles) y con gran capacidad de almacenamiento mediante plaquetas de $1 \mathrm{~Gb}$.

Se determinó, por métodos topográficos convencionales, la línea de frente de la edificación (En este caso, el edificio de la Notaría Internacional), así como la posición de señales de control fijas en la edificación con cintas de papel, para evitar el deterioro de la pintura. También se efectuó el replanteo y medición de la línea de toma fotográfica. Con el objeto de estabilizar la toma, se empleó un trípode provisto de nivel esférico y se procedió a perpendicularizar el eje de toma y determinar su altura. La cámara digital resultó de gran utilidad para fotografiar detalles constructivos, debido a la posibilidad de amplificar mediante zoom digital $(5 x)$ y recortar instantáneamente los sectores de interés (detalles arquitectónicos). Además se cuenta con algunas imágenes de épocas anteriores, que permiten llevar a detalles de autenticidad, la propuesta de intervención a realizar.

Un elemento de suma utilidad que aporta esta cámara es la posibilidad de efectuar una reproducción instantánea de la toma, lo cual elimina la incertidumbre de las cámaras convencionales (debido a iluminación, sombras o incluso a la interposición de vehículos o personas en la línea de toma). También permite eliminar instantáneamente y sin gasto de tiempo y dinero aquellas fotografías de mala calidad y ejecutar las correcciones necesarias.0tra cualidad importante resulta de su capacidad para grabar y reproducir registros sonoros (formato MPEG), con lo cual se pueden identificar objetos o detalles de importancia. Para generar una ortofoto digital, el uso de esta cámara elimina errores de transferencia de la información, a la vez que facilita su almacenamiento en forma compacta.

Las ventajas del procesamiento digital de fotografía arquitectónica han sido descritas por diversos autores, ya sea en su tratamiento de realce de bordes, compensación de contrastes, o en la generación de fotogramas estereoscópicos para generar modelos digitales de los objetos en forma puramente matemática (sin utilización de equipamiento fotogramétrico convencional de gran costo y no disponible para cualquier usuario). Esta tecnología, por otra parte, acorta sensiblemente 
el tiempo de procesamiento (y con ello merma considerablemente su costo) entre la toma y la presentación del resultado. En cuanto a la formación de actitudes y valores profesionales de los estudiantes de Ingeniería, se coincide con la propuesta de educación para la sostenibilidad que hacen Concepción, Rodríguez \& Suárez (2012), en la cual plantean que los estudiantes deben prepararse para un desempeño profesional con un enfoque se sostenibilidad.

Tomando como base el trabajo de Concepción, Rodríguez \& Suárez (2012) sobre la valoración de sostenibilidad de un producto informático, en esta investigación se define la valoración de sostenibilidad de la Fotogrametría Arquitectural Digital, como el proceso de evaluación preventiva de impactos ambientales, socio humanistas, económicos y tecnológicos del procesamiento digital de imágenes, previsibles desde el diseño del proyecto, que favorece su autorregulación, para la satisfacción de la necesidad que resuelve, con un uso racional de recursos y la toma de decisiones apropiadas a las condiciones del contexto.

La valoración de sostenibilidad fue aplicado a la técnica de la FAD en la conservación de bienes inmuebles con valores patrimoniales en el casco histórico de la ciudad de Holguín por el equipo de estudiantes investigadores, lo cual ha favorecido la reflexión acerca de la toma de conciencia de ahorro y uso de recursos con una tecnología adecuada al desarrollo local.

Con los datos así generados, fue posible la reconstrucción del plano de fachada por métodos CAD (generado a partir de la extracción de bordes de la imagen original y corregida geométricamente), que en esta construcción existía paro se hallaba desactualizado por las sucesivas transformaciones a que ha sido sometido el edificio a lo largo del tiempo.

La segunda línea de trabajo corresponde a la carga de la información en un SIG, lo cual conlleva el análisis de la información almacenada y la elaboración de la cartografía respectiva.Los planos utilizan, como base, la cartografía generada por el departamento de Catastro de la ciudad, que permitirá actualizarla a medida que sufra modificaciones.

Para fines de la preservación del monumento, se previó la carga de la información de datos tales como ubicación de redes eléctricas, alcantarillado, agua y la georeferenciación de los hidrantes contra incendios de la ciudad. Los planos así generados permitirán a esta entidad contar con una herramienta de suma utilidad que, inclusive, son de significativa utilidad en la prevención y/o atención de eventos catastróficos, ya que en la actualidad no se cuenta con planos de estas características.

\section{CONCLUSIONES}

- La combinación de estas bases de datos alfanuméricos con imágenes (mosaicos fotográficos de fachadas) o archivos CAD, genera herramientas de trabajo sumamente útiles para la preservación del patrimonio, ya que también permiten evaluar

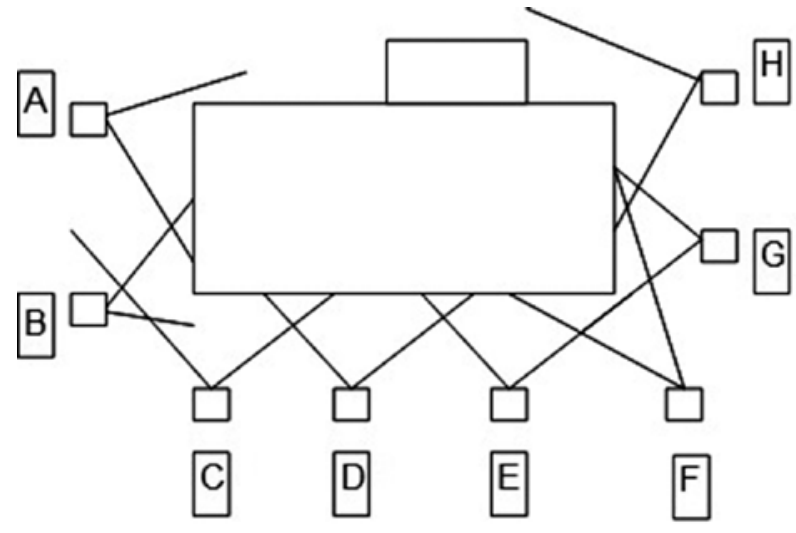

Gráfica 3. Secuencia de tomas fotográficas del objeto

factores de riesgo contra incendios, distancias mínimas de recorrido de bomberos, etc.

- La principal importancia radica en constituir una herramienta valiosa para motivar a organismos gubernamentales, gestores, población en general, para facilitar la realización de acciones de conservación del patrimonio ya que al ser una base de datos permite acceder a la información de cada monumento o edificación de valor histórico o arquitectónico que puede ser de acceso masivo al interconectarse con páginas WEB (como es la tendencia actual de los SIG modernos) ya que publicitar estos datos es una forma fundamental de preservarlos.

- La utilización de equipos de fotografía digital de bajo costo es la ventaja principal de la fotogrametría arquitectónica como la tendencia natural, en forma similar al proceso por el cual esta ha evolucionado hacia sistemas de toma y restitución íntegramente digitales.

- En la investigación se aporta una técnica simple de fotogrametría en combinación con SIG para realizar acciones de conservación del patrimonio edificado del casco histórico de la ciudad de Holguín, Cuba, en el que se propone la cámara digital como herramienta para este tipo de levantamientos, exponiendo sus características más importantes para tal fin, lo que facilita un procedimiento de menor costo del equipamiento $y$ de uso simple que permitan que personal con cierto grado de capacitación realicen el levantamiento.

- El trabajo de investigación relacionado con la búsqueda de alternativas sostenibles de aplicación de la fotogrametrías una vía que ha contribuido a preparara los estudiantes de Ingeniería Civil de la Universidad de Holguín para el desempeño profesional como gestores de conservación del patrimonio arquitectónico. 

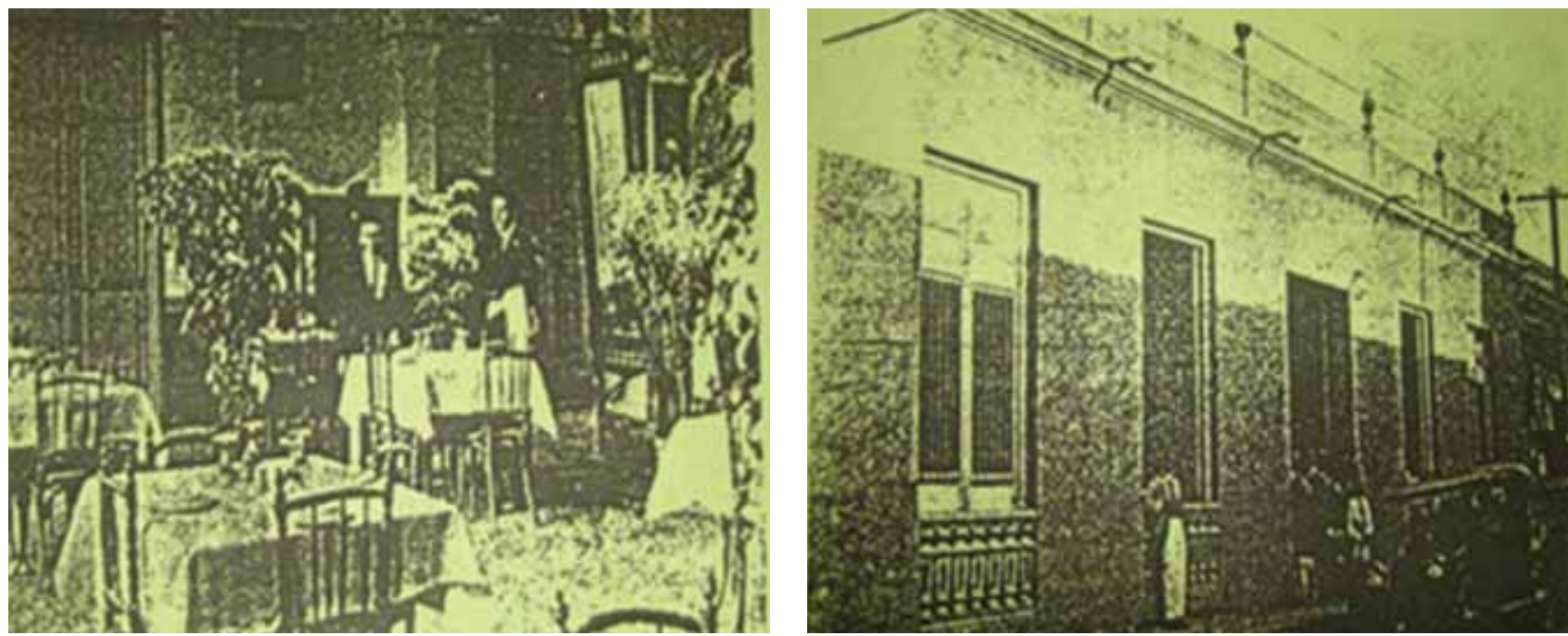

Fuente: Archivo de la Oficina de Patrimonio de la ciudad de guín (2012).

Gráfica 4. Fotografias iniciales que representan las características de la edificación en su inauguración.

- Se deben desarrollar acciones de capacitación técnica y metodológica con los investigadores de todos los municipios del país con patrimonio edificado reconocido y otras personas involucradas en los procesos de conservación, con el fin de fortalecer sus competencias y destrezas en el uso de la fotogrametría arquitectural digital y SIG para el estudio y preservación del patrimonio histórico artístico y arqueológico tangible, empleando la técnica propuesta en esta investigación.

- Contribuir a la sensibilización social de la necesidad de integración de los resultados de la ciencia, la preservación del patrimonio histórico local y el desarrollo óptimo de una industria turística sostenible.

\section{REFERENCIAS BIBLIOGRÁFICAS}

Altrock (2004). Aplicações da fotogrametria arquitetural digital na documentação de edificações históricas - estudo das obras do brigadeiro Joseph da Silva Paes, séc. XVIII. Tesis doctoral. UFSC. Brasil.

Bähr (1991). Procesamiento Digital de Imágenes. Aplicaciones en Fotogrametría y Teledetección. GTZ.

Buill (2007). Amparo Núñez, Juan. J. Rodríguez. Fotogrametría aquitectónica. Ediciones de la Universidad Politécnica de Catalunya, Barcelona.

Cela \& Paredi (1996). Semiótica cartográfica y tecnologías actuales de aplicación.

Cheli (1996). Relevamientos preventivos de nuestro patrimonio.

Claus (1983) Korrelationsrechnung in Stereobildpaaren zur automatischen Gewinnung von digitalen Geländemodellen, Orthophotos und Höhenlinienplänen. DGK, Serie C, Vol. 283:München.
Concepción \& Rodríguez \& Suárez (2012). Educación para la sostenibilidad en docencia de ingeniería. Revista Congreso Universidad Vol 1, No 1 (2012). Editorial universitaria Félix Varela. ISSN: 2306-918X: La habana.

ESRI Inc.: Using ArcView GIS. 1996.

Facon (1993). Processamento e analisede imagens. VI EBAI.

Kreiling (1976). Automatische Herstellung von Höhenmodellen und Orthophotos aus Stereobildern durch digitalle Korrelation. Tesis Doctoral. Univ. Karlsruhe.

Leão de Amorim \& Groetelaars ( 2004). Técnicas de restituição fotogramétricas digitais aplicadas à Arquitetura: um estudo de caso. UFBA: Brasil.

Mena (1992). Cartografía Digital. Desarrollo de software interno. Ed. Ra-ma.

Menéndez (1987). Desperfectos en obras de ingeniería y arquitectura. Ed. MICONS: La Habana.

Navarro \& Rodríguez \&Ávila \&Loch (2010). Conservation projects management by the architectural digital photogrammetry and GIS. 5th International Structural Engineering and Construction Conference, Challenges, Opportunities and Solutions in Structural Engineering and ConstructionGhafoori (Ed.) Pages 683-686 $\odot$ Taylor \& Francis Group, London, ISBN 978-0-415-56809-8 (Hbk), ISBN 978-0203-85992-6 (eBook). University of Nevada: Las Vegas, USA.

Piechel (1985). Verfahren der Stereobild- Korrelation. Digitale Bildverarbeitung H.- P. Bähr. Ed. Wichmann: Karlsruhe.

Santa Cruz. La fotogrametría digital su aplicación en el levantamiento de planos de edificios. Universidad Politécnica de Madrid. Recuperado de: http://www.euatm.upm.es/ santacruz/Fotogrametria/LeccionFotogrametria.pdf (Revisado febrero 2013) 